\title{
Stratospheric isotopic water profiles from a single submillimeter limb scan by TELIS
}

\author{
A. de Lange, J. Landgraf, and R. Hoogeveen \\ SRON Netherlands Institute for Space Research, Sorbonnelaan 2, Utrecht, The Netherlands \\ Received: 30 January 2009 - Published in Atmos. Meas. Tech. Discuss.: 18 March 2009 \\ Revised: 23 July 2009 - Accepted: 23 July 2009 - Published: 5 August 2009
}

\begin{abstract}
Around $490 \mathrm{GHz}$ relatively strong HDO and $\mathrm{H}_{2}{ }^{18} \mathrm{O}$ emission lines can be found in the submillimeter thermal-emission spectrum of the Earth's atmosphere, along with lines of the principal isotopologue of water vapour. These can be used for remote sensing of the rare/principal isotope ratio in the stratosphere. A sensitivity study has been performed for retrieval simulations of water isotopologues from balloon-borne measurements by the limb sounder TELIS (TErahertz and submillimeter LImb Sounder). The study demonstrates the capability of TELIS to determine, from a single limb scan, the profiles for $\mathrm{H}_{2}{ }^{18} \mathrm{O}$ and HDO between $20 \mathrm{~km}$ and $37 \mathrm{~km}$ with a retrieval error of $\approx 3 \%$ and a spatial resolution of $1.5 \mathrm{~km}$, as determined by the width of the averaging kernel. In addition HDO can be retrieved in the range of $10-20 \mathrm{~km}$, albeit with a strongly deteriorated retrieval error. Expected uncertainties in instrumental parameters have only limited impact on the retrieval results.
\end{abstract}

\section{Introduction}

Water is one of the most important species in the Earth's atmosphere, which occurs not only in the humid troposphere but also in the relative arid stratosphere. There it plays a vital role in the radiative budget over a wide spectral range as well as in chemical reactions, both homogeneous and heterogeneous.

The main source of stratospheric water is the influx of humid air from the troposphere in tropical regions. Upon entry into the stratosphere the air crosses the very cold tropopause,

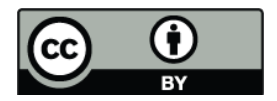

Correspondence to: A. de Lange (a.de.lange@sron.nl) which results in freeze drying of tropospheric air, as was already proposed by Brewer (1949) to explain the stratospheric aridity. Although this explanation has been accepted as the general dehydration process of incoming tropospheric air, the actual mechanisms and locations of the influx have been subject of discussions ever since, and are still not fully resolved (Rosenlof, 2003). Several mechanisms of dehydration have been recognised; convective overshooting (Sherwood and Dessler, 2000), gradual dehydration (Holton and Gettelman, 2001), and ice lofting (Danielsen, 1982; Moyer et al., 1996; Smith et al., 2006). The reaction constants of the various processes differ for different water isotopologues, and therefore, the isotope ratios of water will provide additional insight in the different pathways of dehydration of tropospheric air. In situ measurements by Webster and Heymsfield (2003) have shown that these processes can indeed be discerned by isotope signatures. More recently, Read et al. (2008) have shown how water isotopologue data from satellites can contribute in understanding the roles of convection, mixing, and freeze drying.

A second source of stratospheric water is in situ oxidation of methane and molecular hydrogen. This water is enriched in the rare, heavy isotopes with respect to water transported from the troposphere. As oxidation becomes more important for higher altitudes, this leads to an enrichment of rare isotopes with altitude (Moyer et al., 1996; Johnson et al., 2001; Ridal et al., 2001; Zahn et al., 2006).

Water dynamics become even more complex if chemistry is also accounted for. Water is directly linked to the $\mathrm{HO}_{\mathrm{x}}$ family, and thereby to numerous chemical processes in the stratosphere, including ozone-hole chemistry. Stratospheric ozone is relatively more enriched in ${ }^{17} \mathrm{O}$ than in ${ }^{18} \mathrm{O}$ with respect to other oxygen containing species, dubbed as the ozone anomaly. If stratospheric water vapour shows

Published by Copernicus Publications on behalf of the European Geosciences Union. 
deviations in the relative depletion of the rare-oxygen isotopes with respect to natural abundances, this may give additional information on how water is interconnected to ozone chemistry and how important exchange reactions are (Franz and Röckmann, 2005).

All in all, the stratospheric hydrological cycle is very complex and is still far from understood. To shed light on the manifold of processes of influx, local production, and the various chemical reactions of water, water isotopologues are a useful tool. Vertical stratospheric profiles of water isotopologues can contribute to solving these long-standing issues.

In natural abundance $99.7 \%$ of water consists of the principal isotopologue $\mathrm{H}_{2}{ }^{16} \mathrm{O}$, and hence retrieving concentrations of the rare isotopologues with remote sensing techniques is challenging. Currently operational satellite instruments that provide stratospheric water isotopologue profiles are MIPAS (Steinwagner et al., 2007), Odin (Zelinger et al., 2006; Urban et al., 2007), ACE-FTS (Nassar et al., 2007), and, up to $100 \mathrm{hPa}(\approx 17 \mathrm{~km})$, also TES (Worden et al., 2006). However, due to low $\mathrm{S} / \mathrm{N}$ ratios, averaging is required, at the expense of spatial and/or temporal resolution. In view of the aforementioned science cases, a significant step forward would be the possibility of the retrieval of water isotopologue profiles from a single measurement and thereby improving on spatial and temporal resolution.

With a low spectral resolution spectrometer, the spectral features of the rare isotopologues will most likely be dwarfed by the absorption and emission lines of the principal isotopologue, if not other species. A high resolution spectrometer, with the resolving power of individual lines, is therefore a promising route to obtain the stratospheric isotopic water composition, as the weak lines may be isolated from strong lines.

In this paper the possibilities of isotopic water retrieval from the $480-650 \mathrm{GHz}$ channel of the balloon borne instrument TELIS (TErahertz and submillimeter LImb Sounder) (Hoogeveen et al., 2005; Yagoubov et al., 2008) are investigated by retrieval simulations. The outline of this paper is as follows. In Sects. 2-4 TELIS, the radiative transfer code and the retrieval algorithm are introduced respectively. Then in Sect. 5 profile retrieval of water isotopologues are investigated for the ideal case with perfect knowledge of the instrument. Finally, in Sect. 6, the impact of uncertainties in instrument parameters on these retrievals are studied.

\section{The TELIS instrument}

TELIS is a cryogenic heterodyne balloon sounder developed in a collaboration of three institutes: Deutsches Zentrum für Luft- und Raumfahrt (DLR), Germany, Rutherford Appleton Laboratory (RAL), United Kingdom, and SRON Netherlands Institute for Space Research, the Netherlands. Each institute develops one channel; a miniaturised $500 \mathrm{GHz}$ channel (RAL), a tunable $1.8 \mathrm{THz}$ channel (DLR), and a tunable 480-650 GHz channel (SRON). The instrument either records a $4 \mathrm{GHz}$ spectrum with the $500 \mathrm{GHz}$ channel or records simultaneously two $2 \mathrm{GHz}$ spectra with the $1.8 \mathrm{THz}$ and $480-650 \mathrm{GHz}$ channels. The spectral resolution is $2.2 \mathrm{MHz}$. The latter two channels will both be employed to retrieve profiles of water isotopologues. The presented capacity study pertains to the $480-650 \mathrm{GHz}$ channel by SRON .

TELIS had its maiden flight in June 2008 in Teresina, Brazil. It was mounted on the MIPAS-B2 balloon gondola and flew together with the MIPAS instrument (Michelson Interferometer for Passive Atmospheric Sounding) (FriedlVallon et al., 2004) and mini-DOAS (Differential Optical Absorption Spectroscopy) (Weidner et al., 2005). During this flight all subsystems were separately successfully tested (Yagoubov et al., 2008). The first scientific flight is foreseen in spring 2009 in Kiruna, Sweden.

Heterodyne detection is based on measuring the difference frequency of the atmospheric signal and a reference signal. The reference signal is generated in the so-called local oscillator (LO). It is crucial to keep the LO frequency stable and well defined, and in the $480-650 \mathrm{GHz}$ channel this is achieved by either a phase lock loop or by frequency locking. Both methods result in a different shape of the power spectrum.

Frequency locking results in a Lorentzian lineshape of the LO with a full width half maximum (FWHM) of $2-10 \mathrm{MHz}$, depending on LO frequency. The central frequency, however, may drift by $\approx 2 \mathrm{MHz}$. With the phase lock loop this drift is suppressed and the central frequency is fixed within $1 \mathrm{kHz}$. The lineshape in this case is less well defined, but can be measured separately. Most of the power is generated in a delta-like peak around the locking frequency $(\mathrm{FWHM}<1 \mathrm{kHz})$. A part, however, is stored in two side lobes, located $10-15 \mathrm{MHz}$ from the central peak. It is noted that further signal processing results in an additional uncertainty of the LO frequency of $\approx 1 \mathrm{MHz}$, independent of the chosen locking technique.

The two different locking modes can be selected in-flight and in this paper frequency locking is assumed with an $F W H M=2 \mathrm{MHz}$. Note that the pressure broadening of the atmospheric lines results in linewidths $>10 \mathrm{MHz}$ for altitudes $<37 \mathrm{~km}$ (flight altitude of TELIS), and the actual locking mechanism, and thus the spectral lineshape of the LO is of minor importance.

The atmospheric signal is measured against the absolute difference frequency with respect to the local oscillator. Hence, recorded signal may come from either side of the local oscillator frequency $v_{\mathrm{LO}}$, or sideband. In doublesideband mode no sideband is suppressed, and the measured spectrum is the superposition of two spectra covering the frequency ranges $v_{\mathrm{LO}}+v_{\mathrm{IF}}$ respectively $v_{\mathrm{LO}}-v_{\mathrm{IF}}$, where $v_{\mathrm{IF}}$ is the measured difference frequency, or the intermediate frequency (IF). For the $480-650 \mathrm{GHz}$ channel $v_{\mathrm{IF}}=5-7 \mathrm{GHz}$.

TELIS is currently operating in double-sideband mode. The sideband ratio (SBR) represents the relative magnitude 
of the two sidebands in the recorded spectrum. In the following this ratio is defined as $s=\gamma_{\mathrm{USB}} / \gamma_{\mathrm{LSB}}$, where $\gamma_{\mathrm{USB}}$ and $\gamma_{\text {LSB }}$ are respectively the gain of the upper sideband (USB) and the lower sideband (LSB). The recorded spectrum $I$ is the weighted superposition of the spectra of the two sidebands,

$$
\begin{aligned}
I & =\frac{\gamma_{\mathrm{USB}} I_{\mathrm{USB}}+\gamma_{\mathrm{LSB}} I_{\mathrm{LSB}}}{\gamma_{\mathrm{USB}}+\gamma_{\mathrm{LSB}}} \\
& =\frac{s}{s+1} I_{\mathrm{USB}}+\frac{1}{s+1} I_{\mathrm{LSB}},
\end{aligned}
$$

where $I_{\mathrm{USB}}$ and $I_{\mathrm{LSB}}$ are respectively the spectra of the upper and lower sideband. In this study a sideband ratio of $s=1.0$ is assumed for convenience and hence the weighting factors for both sidebands are 50\%. In Sect. 6.2 deviations in the sideband ratio from unity are addressed.

In the 480-650 GHz channel the receiver antenna, the local oscillator, and the mixer that generates the difference frequency are all situated on a $4 \times 4 \mathrm{~mm}^{2}$ chip, called the Super-conducting Integrated Receiver (SIR). This chip is developed in collaboration with Institute of Radio Engineering and Electronics (IREE), Moscow, and is a major step in the miniaturisation of submillimeter receiver systems. For details we refer to Hoogeveen et al. (2005); Koshelets et al. (2007).

In the 480-650 GHz frequency window, there are a handful relatively strong isotopic water emission lines. Unfortunately, most of these lines are blended by strong ozone or water lines. Notable exceptions are the $\mathrm{H}_{2}{ }^{18} \mathrm{O}$ line at $489.054 \mathrm{GHz}$ and the HDO line at $490.597 \mathrm{GHz}$ which are also the lines probed by the Odin satellite (Urban et al., 2007). It is noted that the HDO line shows a small ozone feature in its wing.

In selecting the local oscillator frequency, one should take care that the lines aimed for do not overlap with lines from the other sideband. With the local oscillator set to respectively $495.040 \mathrm{GHz}$ and $496.880 \mathrm{GHz}$, the $\mathrm{H}_{2}{ }^{18} \mathrm{O}$ and $\mathrm{HDO}$ lines are isolated from lines of the other sideband. Moreover, at these frequencies there is a water line of the principal isotopologue in each spectrum, such that the ratios $\mathrm{H}_{2}{ }^{18} \mathrm{O} / \mathrm{H}_{2}{ }^{16} \mathrm{O}$ and $\mathrm{HDO} / \mathrm{H}_{2}{ }^{16} \mathrm{O}$ can each be determined from a single measurement. It is noted that the main water isotopologue line lies in the same sideband in the case of $\mathrm{H}_{2}{ }^{18} \mathrm{O}$ and in the opposite sideband in case of HDO.

In Fig. 1 typical spectra are depicted for the measurement windows of $\mathrm{HDO}$ and $\mathrm{H}_{2}{ }^{18} \mathrm{O}$ for a tangent height of $27.5 \mathrm{~km}$, meaning the lowest point in altitude along a light path. From these spectra it can be seen that the signal levels of the rare water isotopologues are $\approx 10 \mathrm{~K}$.

The measurement noise per spectral sampling point can be expressed as

$$
\Delta T=\frac{T_{\text {sys }}}{\sqrt{\Delta v t}},
$$
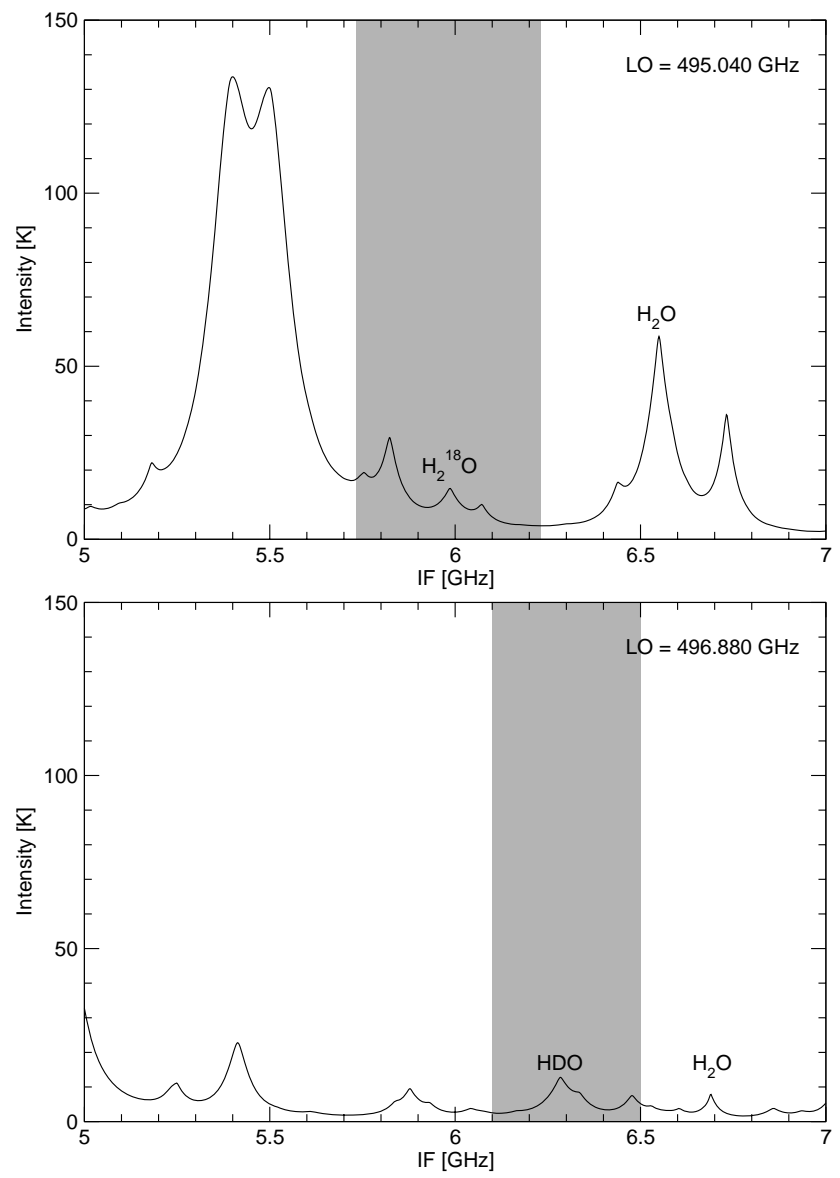

Fig. 1. Noise free spectra as function of difference frequency $v_{\mathrm{IF}}$ for a local oscillator frequency $\nu_{\mathrm{LO}}=495.040 \mathrm{GHz}$ in the upper panel and $v_{\mathrm{LO}}=496.880 \mathrm{GHz}$ in the lower panel. The spectra are for TELIS, recording in double-sideband mode with a sideband ratio of 1.0. The flight altitude is $37 \mathrm{~km}$ and the probed tangent height is $27.5 \mathrm{~km}$. In grey is indicated which part of the spectrum is taken for retrieval of the mentioned molecule.

where $T_{\text {sys }}=250 \mathrm{~K}$ is the noise temperature of the instrument in the frequency range of the rare water isotopologues (Yagoubov et al., 2008), $\Delta \nu=2.2 \mathrm{MHz}$ is the spectral resolution, and $t=1.5 \mathrm{~s}$ is the integration time, which results in an RMS (Root Mean Squares) measurement noise $\Delta T=0.13 \mathrm{~K}$, corresponding to a signal to noise ratio of $\mathrm{S} / \mathrm{N}=10 / 0.13 \approx 75$ for a single spectrum with a tangent point at $27.5 \mathrm{~km}$.

The vertical resolution is determined by the field of view of the instrument in conjunction with the spacing between probed tangent heights and the spectral features in the spectrum. The field of view of the $480-650 \mathrm{GHz}$ channel is almost Gaussian shaped with an FWHM in the vertical direction of 13 arc min at $490 \mathrm{GHz}$ (Yagoubov et al., 2008), which translates to $\approx 1.5 \mathrm{~km}$ at a tangent point of $26 \mathrm{~km}$ which is the middle of the limb sequence. The field of view in the 
horizontal direction is twice as large, but horizontal gradients in the atmosphere are much smaller than vertical gradients and can be neglected on this scale.

The limb sequence for water isotopologue measurements covers the altitude range of $10-36.5 \mathrm{~km}$. Below $20 \mathrm{~km}$ the tangent height spacing is set to $1 \mathrm{~km}$ and above $20 \mathrm{~km}$ to $1.5 \mathrm{~km}$ resulting in a limb sequence of 22 spectra. The rationale behind this is that the range below $20 \mathrm{~km}$ is of relevance for the influx of tropospherical water into the stratosphere. The total time required for a single limb scan will be $\approx 1 \mathrm{~min}$, including radiometric calibration cycles.

\section{Forward model}

The aim of the forward model is to simulate the measurements by TELIS for a given state of the atmosphere and viewing geometry. For this purpose the radiative transfer equation is solved, which gives the intensity $I(v, s)$ of radiation with frequency $v$ at location $s$ in a light path. For a non-scattering atmosphere in local thermal equilibrium the radiative transfer equation can be integrated along the instrument's line of sight

$$
\begin{aligned}
I(\nu, s)= & I\left(\nu, s_{0}\right) \mathrm{e}^{-\tau\left(\nu, s_{0}, s\right)}+ \\
& \int_{s_{0}}^{s} B\left(\nu, T\left(s^{\prime}\right)\right) \kappa\left(\nu, s^{\prime}\right) \mathrm{e}^{-\tau\left(\nu, s^{\prime}, s\right)} d s^{\prime},
\end{aligned}
$$

where $B(T)$ is the Planck function at temperature $T$, and $I\left(\nu, s_{0}\right)$ is the background intensity at the start of the line of sight. The optical density $\tau$ is given by

$\tau\left(\nu, s^{\prime}, s\right)=\int_{s^{\prime}}^{s} \kappa\left(\nu, s^{\prime \prime}\right) d s^{\prime \prime}$,

and the absorption coefficient $\kappa$ is defined as

$\kappa(\nu, s)=\sum_{m} k_{m}(\nu, s) n_{m}$,

with $k_{m}$ the absorption cross section per molecule of molecule $m$ and $n_{m}$ its number density. In general $k_{m}$ is a summation over many lines, each with different central frequency, line strength, and line profile. To account for random, collision-induced, phase shifts the van Vleck-Weisskopf correction is applied to the Voigt line profiles (Vleck and Weisskopf, 1945). The actual profiles are based on line parameters taken from the HITRAN 2004 database (Rothman et al., 2005).

In the $480-650 \mathrm{GHz}$ frequency range water shows some strong emission lines, the most notable around $557 \mathrm{GHz}$. Even far from line centre the wings contribute significantly to the spectrum, acting as an offset or continuum. The calculated line profiles show small discrepancies with the actual line profiles. To account for this, the empirical Liebe model for the continuum is adopted (Liebe, 1989).

The total path integral of the radiative transfer equation (Eq. 3) is numerically solved. This path is slightly curved due to the pressure and temperature dependence of the index of refraction and is included in the model.

To account for instrument characteristics, the modelled atmospheric spectra are first convolved with the instrumental lineshape, as determined by the locking mechanism of the local oscillator. Then the spectra are convolved with the field of view (FOV) of the telescope. Finally the absolute difference frequency with the local oscillator is taken, effectively mapping the two sidebands on top of each other. The latter is true in double sideband mode, but also in single sideband mode if the suppressed sideband still gives rise to residual signal.

\section{Retrieval and regularisation}

With the forward model a spectrum for a specific atmosphere and geometry is calculated that is representative for the TELIS measurement. In the following the forward model will be denoted by $\boldsymbol{F}$ and the discrete set of atmospheric parameters by the vector $\boldsymbol{x}$. A measurement $\boldsymbol{r}$ can be simulated by

$\boldsymbol{r}=\boldsymbol{F}(\boldsymbol{x})+\boldsymbol{e}_{\boldsymbol{y}}$,

where $\boldsymbol{e}_{\boldsymbol{y}}$ is the measurement error including noise and biases.

The aim of retrieval is to invert Eq. (6) to obtain the atmospheric condition $\boldsymbol{x}$ from the measurement $\boldsymbol{r}$. As the forward model is nonlinear a Gauss-Newton iterative scheme is deployed to solve the inversion problem. In every iteration the forward model $\boldsymbol{F}$ is linearised by means of a Taylor expansion around a reference state $\boldsymbol{x}_{i-1}$ and solved for $\boldsymbol{x}_{i}$, where the index $i$ refers to the iteration number. This results in

$\boldsymbol{y}=\mathbf{K} \boldsymbol{x}_{i}+\boldsymbol{e}_{\boldsymbol{y}}$,

where $\mathbf{K}$ is the Jacobian matrix and $\boldsymbol{y}=\boldsymbol{r}-\boldsymbol{F}\left(\boldsymbol{x}_{i-1}\right)+\mathbf{K} \boldsymbol{x}_{i-1}$.

A least squares fit minimises the residue of the modelled spectrum with respect to the measured spectrum,

$\boldsymbol{x}_{\mathrm{lsq}}=\min _{\boldsymbol{x}}\|(\mathbf{K} \boldsymbol{x}-\boldsymbol{y})\|^{2}$.

Within a limb scan each recorded spectrum probes predominately the atmospheric state at a different tangent height, and therefore all spectra contain different information about the atmosphere. At first glance one might therefore believe that a least squares fit with a retrieval grid equal to the probed tangent heights would give good results. However, the atmosphere above the instrument, where no tangent points are possible, may not be neglected in the forward model and will be of influence for the retrieval. Below the lowest tangent point the atmosphere is probed due to the extended field of view. As a result Eq. (8) becomes an ill-posed problem, implying that different atmospheric profiles of temperature, pressure, and molecular concentrations, reproduce the measurement within noise limits. 
The least squares solution will then predominately be determined by the noise on the measurement, rather than the spectral features. To address this problem Tikhonov regularisation (Tikhonov, 1963; Phillips, 1962; Twomey, 1963) is employed in this study.

With Tikhonov regularisation the least squares condition is minimised in addition to a side constraint, sensitive to the retrieval noise. The minimisation equation becomes

$\boldsymbol{x}_{\gamma}=\min _{\boldsymbol{x}}\left(\|(\mathbf{K} \boldsymbol{x}-\boldsymbol{y})\|^{2}+\gamma^{2}\|\mathbf{L} \boldsymbol{x}\|^{2}\right)$

where $\gamma$ is the regularisation parameter, balancing the contributions of the least square solution and the side constraint, and $\mathbf{L}$ is a suitably chosen matrix. In its standard form, the side constraint is the norm of $\boldsymbol{x},\|\boldsymbol{x}\|^{2}$, and subsequently $\mathbf{L}$ is the identity matrix. Other choices of $\mathbf{L}$ include the matrix representation of the $n$th derivative of $\boldsymbol{x}$. In this study, however, Tikhonov regularisation is employed in its standard from.

It is clear that a good choice of the regularisation parameter $\gamma$ is of crucial importance for the solution. It should be chosen large enough that the side constraint filters out noise contributions. However, if $\gamma$ is chosen too large, the least square residual norm will not be minimised anymore, and the spectrum associated with the retrieved vector will depart from the measurement.

A successful approach to set the regularisation parameter $\gamma$ is the use of the so-called L-curve as proposed by Hansen (1992). The L-curve is a parametric plot of the logarithms of the two terms in the minimisation function of Eq. (9), i.e. the norm $\|\boldsymbol{x}\|^{2}$ vs the least squares norm $\|(\mathbf{K} \boldsymbol{x}-\boldsymbol{y})\|^{2}$. For small values of $\gamma$ the least squares residue will be minimised, and the norm will be large. If $\gamma$ is slowly increased, the norm of $\boldsymbol{x}$ will sharply drop while the residual norm will almost stay fixed at its minimal value. This means that the spectrum associated with this state vector $\boldsymbol{x}$ still represents the measurement, while the noise contributions become suppressed. There is a rather well defined point where further increment of $\gamma$ will result in an increase of the least squares norm, indicating a departure from the measurement, without much change in the contribution of the side constraint. This point is the bend in the L-curve and the corresponding $\gamma$ balances the two norms, resulting in a state vector $\boldsymbol{x}_{\gamma}$ that is mostly determined by the atmospheric condition as opposed to the noise on the measurement. We refer to Hansen (1992) for the algorithm to locate this point.

Once the regularisation parameter $\gamma$ is set, the solution can be written as

$\boldsymbol{x}_{\gamma}=\mathbf{D} \boldsymbol{y}$

where $\mathbf{D}$ is the pseudo-inverse of $\mathbf{K}$ or the contribution matrix,

$\mathbf{D}=\left(\mathbf{K}^{\mathrm{T}} \mathbf{K}+\gamma^{2} \mathbf{I}\right)^{-1} \mathbf{K}^{\mathrm{T}} \mathbf{S}_{\boldsymbol{y}}^{-\frac{1}{2}}$ with $\mathbf{S}_{\boldsymbol{y}}$ the measurement noise covariance matrix and $\mathbf{I}$ the unity matrix.

Due to the measurement error, a retrieval noise error is imposed on the retrieved vector. This error propagation is also governed by the contribution matrix,

$\mathbf{S}_{\boldsymbol{x}}=\mathbf{D S}_{\boldsymbol{y}} \mathbf{D}^{\mathrm{T}}$

where $\mathbf{S}_{\boldsymbol{x}}$ is the retrieval noise covariance matrix.

The retrieved vector $\boldsymbol{x}_{\gamma}$ is not an estimate of the true atmospheric state vector $\boldsymbol{x}_{\text {true }}$, but rather a weighted average. By employing Eqs. (7) and (10) the retrieved vector can be expressed as function of the true vector as follows

$\boldsymbol{x}_{\gamma}=\mathbf{A} \boldsymbol{x}_{\text {true }}+\boldsymbol{e}_{\boldsymbol{x}}$,

where $\mathbf{A}=\mathbf{D K}$ is the averaging kernel and $\boldsymbol{e}_{\boldsymbol{x}}=\mathbf{D} \boldsymbol{e}_{\boldsymbol{y}}$ the error in the state vector caused by measurement errors.

It is noted here that $\boldsymbol{x}_{\gamma}$ may contain more than one atmospheric profile and also other parameters to be retrieved. As the norm is used as as side constraint, it may be that this norm is mainly determined by only a subset of these parameters, if these are much larger than others. This is circumvented by a rescaling of the elements of the state vector which is employed in the current study where appropriate.

By setting the regularisation parameter $\gamma$, and thereby the contribution matrix $\mathbf{D}$, a retrieval produces three quantities; the retrieved state vector $\boldsymbol{x}_{\gamma}$, the averaging kernel $\mathbf{A}$, and the covariance matrix $\mathbf{S}_{\boldsymbol{x}}$. In the following the $\boldsymbol{x}_{\gamma}$ is given in units of volume mixing ratio (VMR) and the retrieval error, or the diagonal of the covariance matrix, in percent of the true profile. The averaging kernel is associated with the spatial resolution of the retrieval. To interpret the kernel as a measure of resolution, the FWHM is calculated for every row in kilometres.

\section{Water isotopologue retrieval}

Retrieval simulations have been performed for the rare water isotopologues $\mathrm{H}_{2}{ }^{18} \mathrm{O}$ and $\mathrm{HDO}$ in the $480-650 \mathrm{GHz}$ channel of TELIS. The retrieval results are all for a single limb scan, without further averaging. For this study synthetic spectra are generated with instrument parameters as given in Sect. 2 and are for convenience also listed in Table 1.

Besides the rare isotopologues of water, the species $\mathrm{O}_{3}$, $\mathrm{O}_{2}, \mathrm{H}_{2} \mathrm{O}, \mathrm{N}_{2} \mathrm{O}$, and $\mathrm{ClO}$ are taken into account in the forward model in this study. The atmospheric conditions are set to the AFGL US standard atmosphere (Anderson et al., 1986), with the tropopause of $217 \mathrm{~K}$ at $\approx 12 \mathrm{~km}$. However, Anderson et al. does not provide profiles for different isotopologues. For the current study the profiles for the rare isotopologues are constructed by taking the profile of the principal isotopologue $\mathrm{H}_{2}{ }^{16} \mathrm{O}$ which is then corrected for the natural abundance of the rare isotopologue. It is noted that the profile of HDO may be depleted in the stratosphere by 30-50\% which would lead to lower signal strengths and consequently 

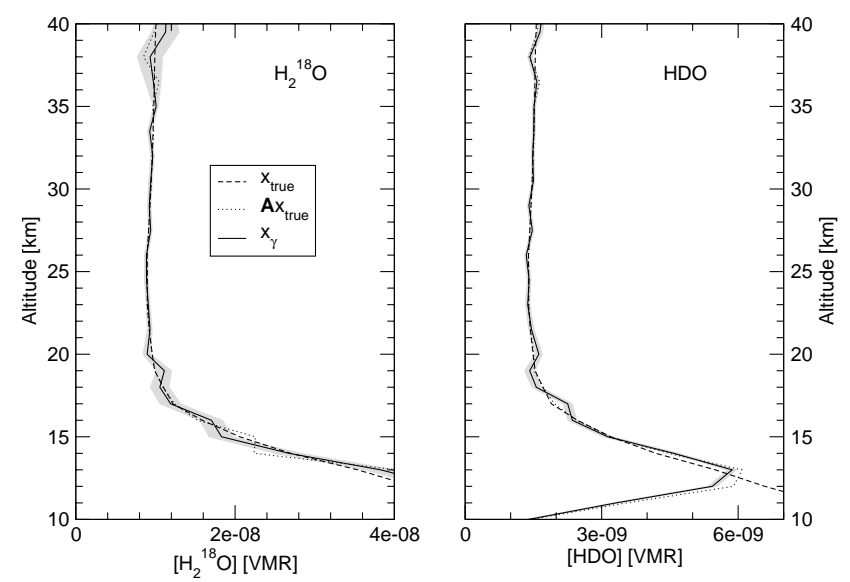

Fig. 2. Typical example of state vectors for $\mathrm{H}_{2}{ }^{18} \mathrm{O}$ (left panel) and HDO (right panel) for TELIS. The retrieved profile (solid) is shown along with the retrieval error (grey), the true profile (dashed), and $\mathbf{A} \boldsymbol{x}_{\text {true }}$ (dotted). The results are obtained from a single limb sequence with 22 spectra recorded at different tangent points and an integration time of $1.5 \mathrm{~s}$ with perfect knowledge of the instrument parameters.

to higher retrieval errors. This depletion is a function of altitude and lessens with increasing altitude due to methane oxidation. The depletion of stratospheric $\mathrm{H}_{2}{ }^{18} \mathrm{O}$ is less than $10 \%$. Examples of noise-free simulated spectra are depicted in Fig. 1 for both $\mathrm{HDO}$ and $\mathrm{H}_{2}{ }^{18} \mathrm{O}$. The parts indicated in grey are used for retrieval.

In this study the temperature, pressure, $\mathrm{O}_{2}, \mathrm{~N}_{2} \mathrm{O}$, and $\mathrm{ClO}$ profiles are assumed to be known and set to their a priori values.

The $\mathrm{H}_{2}{ }^{18} \mathrm{O}$ line occurs in the wing of a strong water line (just outside the spectrum of Fig. 1) acting as a large baseline offset for low tangent heights. Therefore, $\mathrm{H}_{2} \mathrm{O}$ is retrieved simultaneously with $\mathrm{H}_{2}{ }^{18} \mathrm{O}$. The purpose of this retrieval is not to get a proper water profile, but rather to account for the offset such that its influence on the $\mathrm{H}_{2}{ }^{18} \mathrm{O}$ retrieval is minimised. For this retrieval, the $\mathrm{O}_{3}$ profile is set to the a priori values.

In the wing of the HDO line a $\mathrm{O}_{3}$ feature resides. To investigate how well $\mathrm{HDO}$ can be retrieved without a priori knowledge of ozone, the $\mathrm{O}_{3}$ profile is retrieved simultaneously with $\mathrm{HDO}$. For $\mathrm{HDO}$ retrieval, the $\mathrm{H}_{2} \mathrm{O}$ profile is set to the a priori value.

In Fig. 2 the retrieved state vector (solid line) with its retrieval error (grey area), the true profile (dashed line), and $\mathbf{A} \boldsymbol{x}_{\text {true }}$ (dotted line) are plotted in Fig. 2 for $\mathrm{H}_{2}{ }^{18} \mathrm{O}$ (left panel) and HDO (right panel) for the case that only receiver noise is considered. As first guess, the true state vector is taken with a scaling factor. The scaling factor has no influence on the retrieval results in the range of $10 \%$ to the upper limit of $400 \%$. In Fig. 3 the retrieval error, the averaging kernel and its FWHM are shown for both isotopologues. It can
Table 1. Instrument parameters used for the generation of synthetic spectra. LO stands for local oscillator, FWHM for full width half maximum, and FOV for field of view.

\begin{tabular}{ll}
\hline LO frequency & $495.040 \mathrm{GHz}\left(\mathrm{H}_{2}{ }^{18} \mathrm{O}\right)$ \\
& $496.880 \mathrm{GHz}(\mathrm{HDO})$ \\
LO shape & Lorentzian \\
LO FWHM & $2 \mathrm{MHz}$ \\
Sideband ratio & 1.0 \\
FOV FWHM & 13 arc minutes \\
Integration time & $1.5 \mathrm{~seconds}$ \\
RMS noise & $0.13 \mathrm{~K}$ \\
Limb sequence & $10-20 \mathrm{~km} @ 1.0 \mathrm{~km}$ \\
& $20-36.5 \mathrm{~km} @ 1.5 \mathrm{~km}$ \\
Flight altitude & $37 \mathrm{~km}$ \\
\hline
\end{tabular}

be seen that the averaging kernel peaks to 1.0 over an altitude range of $15-35 \mathrm{~km}$ in the case of $\mathrm{H}_{2}{ }^{18} \mathrm{O}$ and $20-35 \mathrm{~km}$ in the case of HDO resulting in an FWHM equal to the tangent spacing, i.e. $1.5 \mathrm{~km}$ above $20 \mathrm{~km}$ and $1.0 \mathrm{~km}$ below. The retrieval error is $2-3 \%$ over a wide range of altitudes, peaking at $17-19 \mathrm{~km}$ at values of $6 \%$ and $11 \%$ for respectively HDO and $\mathrm{H}_{2}{ }^{18} \mathrm{O}$. For HDO there is a clear correlation between the broadening of the averaging kernel, effectively averaging the profiles over a wider range of altitudes, and the decrease of the retrieval error for the lower part of the limb scan. It is noted that the absolute retrieval error increases with decreasing altitude below $20 \mathrm{~km}$ for both isotopologues as both the volume mixing ratio and the air density increase.

Above $37 \mathrm{~km}$ the retrieval error increases for both $\mathrm{H}_{2}{ }^{18} \mathrm{O}$ and HDO in conjunction with a broadening of the averaging kernel as information in the spectra is limited for these altitudes. This is because no tangent heights are possible above the flight altitude of $37 \mathrm{~km}$. Although the radiative transfer of this altitude range is of importance for the retrieval at lower altitudes, the actual retrieved values are of limited value only.

Another aspect to consider are cross-correlations between different profiles. In Fig. 3 only the part of the averaging kernel is depicted that links the retrieved profile to the true profile of that particular species. The cross-averaging kernel links the retrieved profile to the true profile of another species, i.e. $\mathrm{H}_{2} \mathrm{O}$ in case of $\mathrm{H}_{2}{ }^{18} \mathrm{O}$ retrieval and $\mathrm{O}_{3}$ in case of HDO. Ideally these values are close to zero, which would indicate that the retrieved isotopic water profile is not mixed with water, respectively ozone. For HDO, this is indeed the case and the retrieved profile is not intermingled with the ozone profile. For $\mathrm{H}_{2}{ }^{18} \mathrm{O}$, however, the cross-averaging kernel departs from zero in the range $10-20 \mathrm{~km}$, as is illustrated in the left panel of Fig. 4. This means that for $\mathrm{H}_{2}{ }^{18} \mathrm{O}$ significant amounts of $\mathrm{H}_{2} \mathrm{O}$ is mixed into the isotopic water profile below $20 \mathrm{~km}$. In the right panel the contributions of the averaging kernel and the cross-averaging kernel to the retrieved $\mathrm{H}_{2}{ }^{18} \mathrm{O}$ profile are both shown. Below $20 \mathrm{~km}$ the contribution of the cross-averaging kernel increases with decreasing 

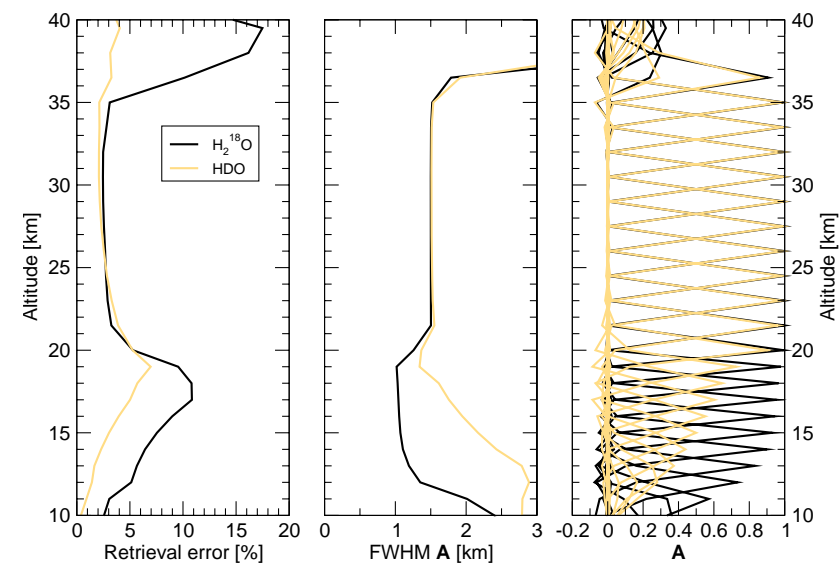

Fig. 3. The diagonal elements of the retrieved covariance matrices (left panel), the widths of the retrieved averaging kernels (centre panel), and the averaging kernels (right panel) for a typical HDO retrieval, respectively, $\mathrm{H}_{2}{ }^{18} \mathrm{O}$ retrieval with TELIS. In Fig. 2 the retrieved state vectors are illustrated.

altitude, contaminating the retrieved isotopic water profile and hampering a straightforward interpretation.

In conclusion the retrieval for HDO can be done over the whole $10-37 \mathrm{~km}$ altitude range of the limb sequence, with a retrieval error of $1-6 \%$ in the undepleted case. In case HDO is depleted the retrieval error ranges from $\approx 2-8 \%$. The spatial resolution for HDO, as determined by the averaging kernel widths, is $1.5 \mathrm{~km}$ above $20 \mathrm{~km}$ and below $20 \mathrm{~km} \mathrm{1.5-}$ $2.5 \mathrm{~km}$.

For $\mathrm{H}_{2}{ }^{18} \mathrm{O}$ the retrieval results in an uncontaminated profile above $20 \mathrm{~km}$, with a retrieval error of $\approx 3 \%$ and an averaging kernel FWHM of $1.5 \mathrm{~km}$. The retrieval below $20 \mathrm{~km}$ is hampered by a strong water line and the retrieval results are hard to interpret as the profile becomes mixed with the profile of $\mathrm{H}_{2} \mathrm{O}$.

\subsection{Temperature error propagation}

The assumed temperature profile has only limited accuracy and may therefore have an impact on the retrieval error. The temperature profile will be taken from the analysis data by the ECMWF (European Centre for Medium-Range Weather Forecasts). The accuracies for March 2009 in Northern Scandinavia is on the order of $1 \mathrm{~K}$ up to the maximum validated pressure level of 10 mbar which corresponds to an altitude of $\approx 32 \mathrm{~km}$ (K. Fielding, private communication, eCMWF, 2009). A slight increase can be discerned above $\approx 27 \mathrm{~km}$. The accuracies are in the following analysis set to $1 \mathrm{~K}$ for altitudes up to $27 \mathrm{~km}$ and then a linear increase with altitude to $2 \mathrm{~K}$ at $46 \mathrm{~km}$. To assess the error propagation of this uncertainty onto the retrieved rare water isotopologue profiles the following formula is employed:

$\mathbf{S}_{\boldsymbol{x}}=\mathbf{D K}_{T} \mathbf{S}_{T} \mathbf{K}_{T}^{\mathrm{T}} \mathbf{D}^{\mathrm{T}}$
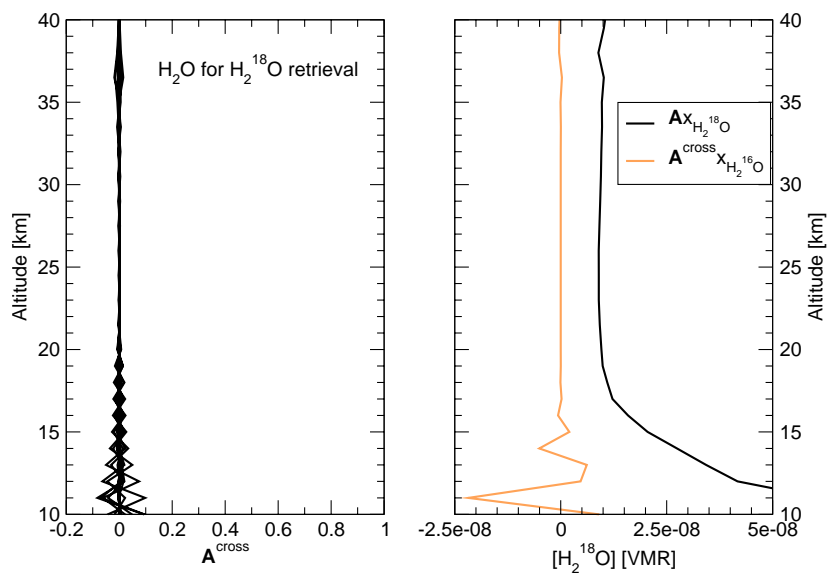

Fig. 4. The cross-averaging kernel of $\mathrm{H}_{2} \mathrm{O}$ acting on $\mathrm{H}_{2}{ }^{18} \mathrm{O}$ is shown in the left panel. In the right panel $\mathbf{A} \boldsymbol{x}_{\text {true }}$ is depicted for both contributions of $\mathrm{H}_{2} \mathrm{O}$ (orange line) and $\mathrm{H}_{2}{ }^{18} \mathrm{O}$ (black line).

with $\mathbf{S}_{T}$ the ECMWF temperature covariance matrix, $\mathbf{D}$ the contribution matrix, $\mathbf{K}_{T}$ the Jacobian of the temperature, and $\mathbf{S}_{\boldsymbol{x}}$ the covariance matrix of the retrieved state vector (Rodgers, 2000). The result of this analysis is depicted in Fig. 5. As can be seen, the error is in the range of the tangent heights only $\approx 0.1 \%$, which is less than the retrieval error (see Fig. 3) and is therefore of only minor consequence. Alternatively, the temperature profile may be determined from the measurements from the ${ }^{16} \mathrm{O}^{18} \mathrm{O}$ line at $508.996 \mathrm{GHz}$.

\section{Sensitivity to instrumental parameters}

In the results presented above, perfect knowledge of instrumental parameters is assumed. Unlike with synthetic data this is obviously not the case with actual measurements. A sensitivity study has been performed to see how imperfect knowledge influences the retrieval results. The considered error sources are pointing knowledge, sideband ratio, LO frequency, field of view, and the instrumental lineshape.

\subsection{Pointing}

The uncertainty in the pointing of the telescope contributes generally significantly to the error budget in limb retrieval. It is therefore mandatory to inspect its influence on the retrieval. Currently, TELIS receives its pointing information from the MIPAS instrument, which is equipped with a dedicated attitude and heading reference system (AHRS) (FriedlVallon et al., 2004). The stability of this system is much better than 1 arc minute. However, both TELIS and MIPAS are connected by several springs to the gondola frame and it remains untested how these springs will influence the actual pointing with respect to the received pointing information. It is estimated that this pointing mismatch is up to $10 \mathrm{arc} \mathrm{min}$. 

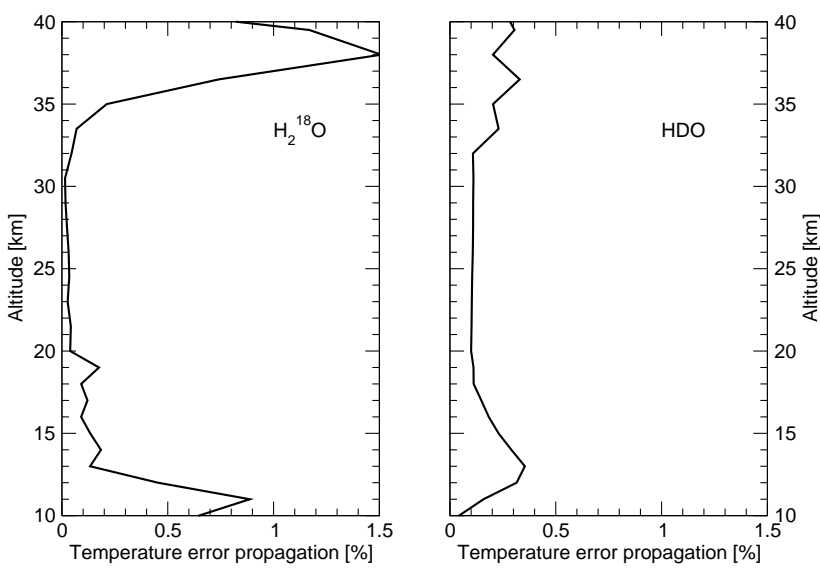

Fig. 5. The error propagation of the limited accuracy on the temperature on the retrieved $\mathrm{HDO}$ and $\mathrm{H}_{2}{ }^{18} \mathrm{O}$ profiles is depicted. The error is given in $\%$ relative to the true state vector and can thus directly be compared against the retrieval error.

It is investigated what the effect of a pointing error is for both a systematic offset and a random pointing error.

If pointing is not accounted for, the spectra corresponding to the retrieved profiles show systematic residuals with respect to the measurement, implying that pointing information is contained in the measurements. In contrast to the retrieval in Sect. 5, in the following the pointing angle is retrieved for each spectrum in the limb sequence in addition to the molecular profiles.

The retrieval results for HDO for a systematic pointing offset are depicted in Fig. 6. In this figure, the dashed black line refers to the retrieval in Sect. 5, i.e. a retrieval with perfect knowledge of the instrument and no instrumental parameters are retrieved. The solid black line in the left and centre panels corresponds to the case where no pointing offset is applied, but nevertheless pointing information is retrieved. In the range $20-35 \mathrm{~km}$ the dashed and solid black lines coincide in the centre panel, indicating that the averaging kernel in this altitude range is virtually unaffected. Below $20 \mathrm{~km}$, however, the averaging kernel narrows when pointing information is retrieved. This corresponds to the observed increase in the retrieval error in this altitude range. The increase in retrieval error is by a factor of 6 , up to $35 \%$.

This is due to the fact that the HDO line is pressure broadened at low altitudes. A pointing error results in first order, at low altitudes, in an intensity shift of the spectrum. The sensitivity of the spectrum to the HDO profile and the pointing angle show therefore in both cases broad spectral features and no distinction can be made anymore between contributions from one or the other. The addition of extra parameters to the state vector results then in the increase of the retrieval error.

When a pointing offset is applied, the retrieval error and averaging kernel below $20 \mathrm{~km}$ are virtually unaffected with
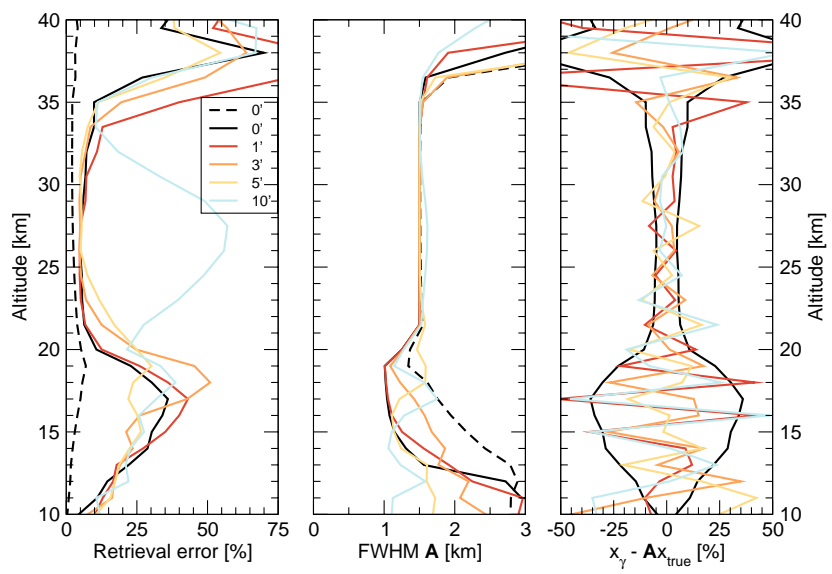

Fig. 6. The retrieval error, the FWHM of the averaging kernels, and $\boldsymbol{x}_{\gamma}-\mathbf{A} \boldsymbol{x}_{\text {true }}$ for HDO retrieval with a systematic pointing error. For reference, the retrieval results with no offset is illustrated as well for the case that pointing information is not retrieved (dashed black line) and that pointing information is simultaneously retrieved (solid black line). The pointing offset covers 1-10 arc min, which corresponds to a shift of the tangent height of $200 \mathrm{~m}$ to $2 \mathrm{~km}$ for the lowest tangent points. In the right panel the solid black line refers to the retrieval error in case of no pointing offset.

respect to the retrieval without an offset. In fact, this is true over a wide range of pointing offsets. Above $20 \mathrm{~km}$, however, the retrieval error increases, with increasing pointing error. For 3 arc min the retrieval error is still comparable to a retrieval with perfect instrument knowledge for $23 \mathrm{~km}$ and above, but below $23 \mathrm{~km}$ the error becomes significantly larger. This can be explained by the fact that a pointing offset corresponds to an increasing offset in kilometers at tangent point for decreasing altitude. The impact on the retrieval is therefore highest for the lowest tangent heights. For increasing pointing offsets, the affected range extends to higher altitudes. For an offset of $10 \mathrm{arc}$ min, the retrieval error is deteriorated over the whole altitude range with respect to the retrieval without pointing offset.

Apart from the retrieval error, of a stochastic nature, a systematic error to the retrieved profile may be induced by a pointing offset. This can be investigated by comparing the retrieved profile with the smoothed true profile, or $\boldsymbol{x}_{\gamma}-\mathbf{A} \boldsymbol{x}_{\text {true }}$. This effect is shown in the right panel of Fig. 6. The solid black line refers to the retrieval error without pointing offset. As can be seen, no systematic error is introduced over the whole altitude range, even up to a pointing error of 10 arc min.

In conclusion, HDO retrieval is feasible even for the most severe pointing offsets. The retrieval error, however, becomes large below $20 \mathrm{~km}$, such that a retrieval from a single limb sequence will have only limited information in this altitude range. 
The retrieval results for $\mathrm{H}_{2}{ }^{18} \mathrm{O}$ for a systematic pointing offset are depicted in Fig. 7. Above $20 \mathrm{~km}$ the averaging kernel is unaffected by a pointing error, as was also the case with HDO. For $\mathrm{H}_{2}{ }^{18} \mathrm{O}$, however, the retrieval error seems to be slightly higher than for HDO, with the exception of the largest offset of 10 arc min. When no pointing offset is applied, the averaging kernels of the retrievals with or without pointing information, are very similar. This is in contrast to HDO where a narrowing is observed below $20 \mathrm{~km}$. When a pointing offset is applied, the averaging kernels show some variation below $20 \mathrm{~km}$, which is governed by noise rather than systematic effects, as is the case with HDO. The retrieval errors are comparable to the retrieval errors of HDO. In contrast to HDO, however, is the observation of large systematic errors in the $\mathrm{H}_{2}{ }^{18} \mathrm{O}$ profile below $20 \mathrm{~km}$ (not shown). This is, however, of minor importance as the retrievals in this altitude range are ambiguous even for the case of perfect instrument knowledge as is explained in Sect. 5.

In the above, only a systematic pointing offset was investigated. The results for a random pointing error for each spectrum in the limb sequence, however, are virtually the same. This is due to the implementation of the pointing retrieval. A pointing offset is not retrieved as a single parameter, but is retrieved separately for each spectrum in the limb sequence. The rationale behind this is that it is anticipated that the actual pointing angles of real measurements will show a general offset with a random component superimposed due to the springs by which TELIS is attached to the gondola, and hence, it must be retrieved for every spectrum separately. The retrieval results are therefore very comparable for a systematic offset or an offset with a random nature.

In conclusion, if there is an uncertainty in the pointing information, both $\mathrm{HDO}$ and $\mathrm{H}_{2}{ }^{18} \mathrm{O}$ can still be retrieved above $20 \mathrm{~km}$, up to a pointing error of about 5 arc min. For HDO the retrieval is also possible below $20 \mathrm{~km}$, albeit that the retrieval error peaks to $\approx 35 \%$. In this range $\mathrm{H}_{2}{ }^{18} \mathrm{O}$ retrieval is hampered due to $\mathrm{H}_{2} \mathrm{O}$ interference, as is explained in Sect. 5

\subsection{Sideband ratio}

An error in the knowledge of the sideband ratio (SBR) leads to a systematic shift in the contributions of the two sideband spectra, and may lead to a systematic over- or underestimation of the retrieved species. This can be seen as follows. Suppose that the relative error in the knowledge of this ratio is $\epsilon$, then Eq. (1) becomes

$I=\frac{s(1+\epsilon)}{s(1+\epsilon)+1} I_{\mathrm{USB}}+\frac{1}{s(1+\epsilon)+1} I_{\mathrm{LSB}}$,

which, by neglecting higher order terms in $\epsilon$, can be rewritten as

$$
\begin{aligned}
I \approx & \frac{s}{s+1}\left(1+\frac{\epsilon}{s+1}\right) I_{\mathrm{USB}}+ \\
& \frac{1}{s+1}\left(1-\frac{\epsilon}{s+1}\right) I_{\mathrm{LSB}}
\end{aligned}
$$
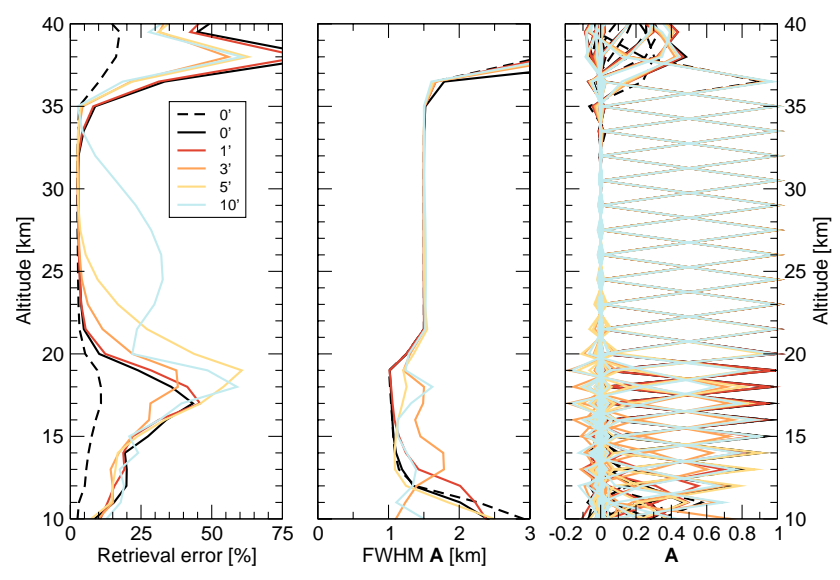

Fig. 7. The retrieval error, the widths of the averaging kernels, and the averaging kernels for $\mathrm{H}_{2}{ }^{18} \mathrm{O}$ retrieval with a systematic pointing error. For reference, the retrieval results with no offset is illustrated as well for the case that pointing information is not retrieved (dashed black line) and that pointing information is simultaneously retrieved (solid black line). The pointing offset covers 1-10 arc min, which corresponds to a shift of the tangent height of $200 \mathrm{~m}$ to $2 \mathrm{~km}$ for the lowest tangent points.

which is of similar form as Eq. (1) albeit with an extra scaling factor for both sidebands. From these equations it can be seen that an error in the knowledge of the sideband ratio will lead to erroneous contributions of the spectra of the two sidebands. To compensate for this effect, the retrieval algorithm will alter the state vector, and thus the profile, effectively nullifying these scaling factors.

The HDO line occurs in this study in the lower sideband and from Eq. (16) it is then expected that an overestimation of the sideband ratio by a factor $\epsilon$ will lead to an underestimation of the retrieved profile by a factor of $\frac{\epsilon}{2}$, for the assumed sideband ratio $s=1.0$. The retrieval results on this systematic effect are shown in Fig. 8. Indeed, for small values of $\epsilon$ the systematic underestimation of the HDO profile is $\frac{\epsilon}{2}$. Above $20 \mathrm{~km}$ the systematic error in the HDO profile is smaller than the retrieval error if $\epsilon<0.06$, and below $20 \mathrm{~km}$ if $\epsilon<0.12$.

If one or more lines of a single species is present in both sidebands, the sideband ratio may be retrieved assuming a stable SBR over the frequency interval. For $\mathrm{H}_{2}{ }^{18} \mathrm{O}$ retrieval this is the case; $\mathrm{O}_{3}$ shows spectral features from both sidebands in the retrieval window. The retrieval of the sideband ratio is of no consequence for the $\mathrm{H}_{2}{ }^{18} \mathrm{O}$ retrieval (not depicted). If the error $\epsilon$ is set to zero, the retrieval errors and the averaging kernels are almost identical whether the SBR is retrieved or not. In fact, the retrieval results do not show significant change over the wide range of uncertainties in the sideband ratio of $\epsilon=0.0-0.86$. This, however, assumes perfect knowledge of $\mathrm{O}_{3}$.

In Fig. 9 it is shown how the retrieval of the sideband ratio influences the retrieval of the $\mathrm{H}_{2}{ }^{18} \mathrm{O}$ profile retrieval if ozone 

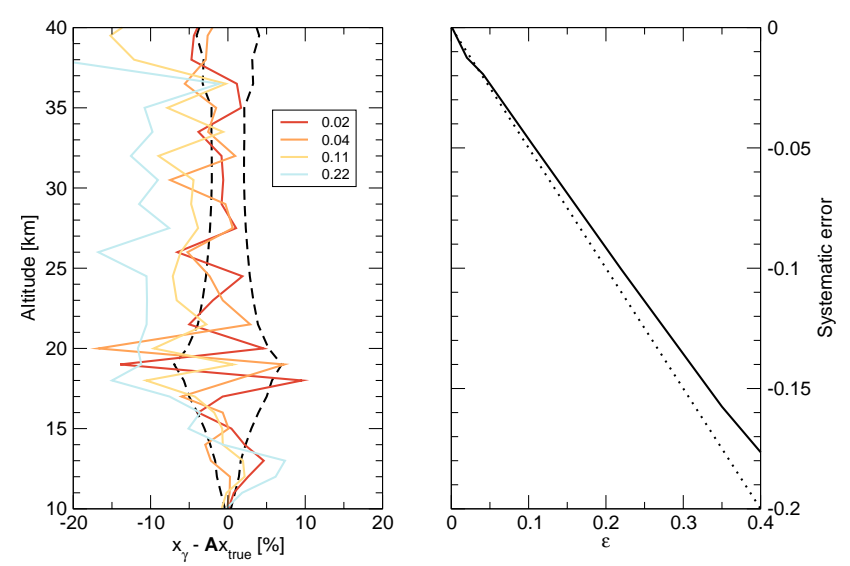

Fig. 8. The systematic error on the retrieved HDO profile induced by an error $\epsilon$ in the sideband ratio. In the right panel the average values of the altitude range $20-35 \mathrm{~km}$ are plotted (solid line). The dotted line corresponds to $-\epsilon / 2$.

is retrieved as well. Between 20 and $35 \mathrm{~km}$, the retrieval of the sideband ratio in conjunction with ozone, leaves the retrieved profile unaffected. Only below $20 \mathrm{~km}$ the averaging kernel broadens and correspondingly the retrieval error decreases, but as explained in Sect. 5 this range is of limited interest only, due to the water interference.

A first measurement of the sideband ratio at $625 \mathrm{GHz}$ showed a variation in the sideband ratio of 1.2-1.3 over $2 \mathrm{GHz}$. To see how robust the retrieval is for a spectrally nonconstant sideband ratio, the observed variation in the sideband ratio is modelled. This is done as follows; $\epsilon$ increases from 0.2 to 0.3 over the IF frequency range $v_{\mathrm{IF}}=5-7 \mathrm{GHz}$. Moreover, a ripple with a frequency of $\approx 600 \mathrm{MHz}$ and an amplitude of 0.03 is super-imposed on this slope. Even in this case the retrieval results for $\mathrm{H}_{2}{ }^{18} \mathrm{O}$ are virtually identical to the retrieval with perfect knowledge on the sideband ratio.

For all altitudes and over the whole range of ratios $\boldsymbol{x}_{\gamma}-\mathbf{A} \boldsymbol{x}_{\text {true }}$ does not show significant deviations from zero for $\mathrm{H}_{2}{ }^{18} \mathrm{O}$ (not shown), i.e. an uncertainty in the sideband ratio causes no systematic effect on the $\mathrm{H}_{2}{ }^{18} \mathrm{O}$ profile. This indicates that the sideband ratio can be retrieved to such an extent that the retrieval of $\mathrm{H}_{2}{ }^{18} \mathrm{O}$ is virtually unaffected.

Unfortunately, in the HDO retrieval window no lines of a single species are available in both sidebands. Ozone lines, however, do occur in the $2 \mathrm{GHz}$ HDO measurement window. These lines may be used to retrieve a sideband ratio, albeit at a slightly different frequency. The only measurement of the sideband ratio at $625 \mathrm{GHz}$ showed a variation in the sideband ratio of less than $5 \%$ over $2 \mathrm{GHz}$. This would imply a systematic error in the retrieved profile of less than $2.5 \%$. Another option is to take the sideband ratio of the $\mathrm{H}_{2}{ }^{18} \mathrm{O}$ measurement window as the $\mathrm{LO}$ frequencies of the measurement windows of the two water isotopologues differ by less than $2 \mathrm{GHz}$, giving rise to a similar systematic error in the
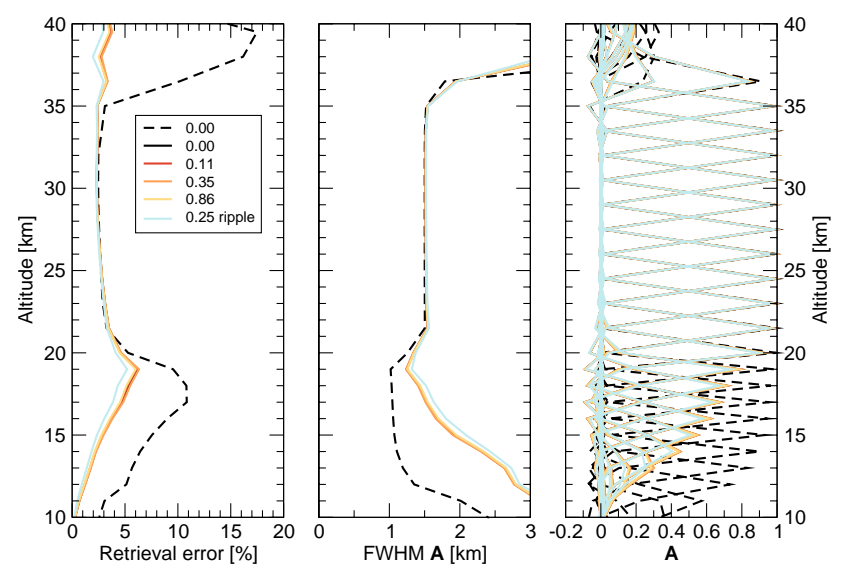

Fig. 9. Retrieval results for $\mathrm{H}_{2}{ }^{18} \mathrm{O}$ with an error $\epsilon$ in the sideband ratio. For reference the results for a retrieval with a known sideband ratio is illustrated as well in case this ratio is not retrieved (dashed line) and in case the ratio is retrieved (solid black line). Note that in the latter case the line is obscured by the other results, indicating that an uncertainty in the knowledge of the sideband ratio does not influence the retrieval. In addition to the sideband ratio is the ozone profile retrieved. The grey line refers to a situation where the sideband ratio varies over the simulated spectrum over a range of $1.2-1.3$ with an average value of 1.25 .

retrieved HDO profile. The actual sideband ratio in all measurement windows are to be determined.

It is noted that for water dynamic studies, the ratio rare isotopologue/main isotopologue is of importance. If the water profile is taken from the rare isotopologue measurement windows, then the effect of an error in the sideband ratio may influence this ratio. In case of $\mathrm{H}_{2}{ }^{18} \mathrm{O}$ both isotopologues reside in the same sideband and systematic effects of an erroneous sideband ratio on the isotope ratio will cancel, even if the sideband ratio is not retrieved. In case of HDO, however, both isotopologues are in different sidebands and an error in the sideband ratio will cause a systematic error in the isotope ratio. For a systematic error of $2.5 \%$ in the HDO profile, the systematic error is $5 \%$ in the $\mathrm{HDO} / \mathrm{H}_{2} \mathrm{O}$ ratio.

In conclusion, above $20 \mathrm{~km}$ an error in the sideband ratio does not affect the profile retrieval of $\mathrm{H}_{2}{ }^{18} \mathrm{O}$ as the sideband ratio can be retrieved. For HDO, matters are somewhat more complicated as the sideband ratio may not be retrieved directly from spectral features in the retrieval window. It remains to be seen as to what accuracy the sideband ratio may be determined in this measurement window, but uncertainties of a few percent may already cause biases in the retrieved HDO profile. Currently, no variations in the sideband ratio larger than $5 \%$ are observed over a bandwidth of $2 \mathrm{GHz}$, which would imply a systematic error of $2.5 \%$ in the retrieved HDO profile. The retrieval error for HDO and the averaging kernel are not influenced by an error in the sideband ratio. 


\subsection{LO frequency}

The LO frequency has an intrinsic instability of $1 \mathrm{MHz}$ due to signal processing. In frequency locking mode the central frequency shows an additional uncertainty of $\approx 2 \mathrm{MHz}$. In Fig. 10 it can be seen that shifts up to $3 \mathrm{MHz}$ do not influence the retrieval of the $\mathrm{H}_{2}{ }^{18} \mathrm{O}$ profile, when the LO frequency is retrieved as well. If the uncertainty in LO frequency increases to $5 \mathrm{MHz}$, the averaging kernel broadens somewhat below $20 \mathrm{~km}$, and in conjunction the retrieval error decreases. No systematic errors are observed at altitudes up to flight altitude. At $40 \mathrm{~km}$, however, a systematic error of $+25 \%$ can be observed (not depicted), which is more than the retrieval error of $15 \%$ at that altitude. This altitude lies in the range where no tangent points are possible, and is only of minor importance.

For HDO similar retrieval results are obtained.

Hence, uncertainties in the exact LO frequency are not relevant for isotopic water retrievals with TELIS.

\subsection{Field of view and instrumental lineshape}

The width of the field of view is known within several percent. Simulations show that the importance of this uncertainty becomes only relevant if this value is larger than $10 \%$, and then only in a slight systematic bias below $15 \mathrm{~km}$ of less than $1 \%$. This is true even for the case where the field of view width is not retrieved, and hence this error is of no relevance for isotopic water retrieval with TELIS. An asymmetry in the field of view, may in first order be interpreted as a pointing offset which is discussed in Sect. 6.1.

The width of the instrumental lineshape depends on the locking mechanism of the LO frequency, but is in both cases much narrower than the pressure broadened atmospheric lines. It is therefore expected that the width of this lineshape is only of minor importance. Indeed, an uncertainty of even $50 \%$ does not significantly influence the retrievals, also if this width is kept fixed and is not retrieved. An asymmetry in the instrumental lineshape, may in first order be interpreted as an LO frequency shift which is discussed in Sect. 6.3.

\section{Conclusions}

This paper demonstrates the capability of the $480-650 \mathrm{GHz}$ channel of the TELIS instrument to retrieve profiles for $\mathrm{H}_{2}{ }^{18} \mathrm{O}$ and $\mathrm{HDO}$ from one single limb scan with an integration time of only $1.5 \mathrm{~s}$ per spectrum. This enables water isotopologue profile retrieval at a high spatial/temporal resolution in contrast to current operational platforms.

For both isotopologues the retrieval error is typically $3 \%$ in the altitude range of $20-37 \mathrm{~km}$ and the averaging kernel width is $\approx 1.5 \mathrm{~km}$, corresponding to the altitude step between two consecutive tangent heights in the limb scan and to the extension of the field of view at tangent point. This is for the undepleted case. For HDO depletions of $30-50 \%$ can be
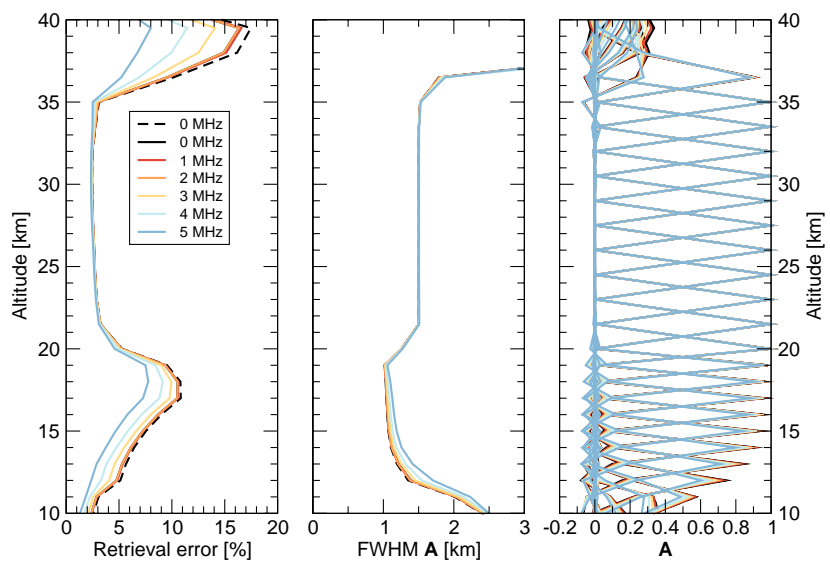

Fig. 10. Retrieval results for $\mathrm{H}_{2}{ }^{18} \mathrm{O}$ with an error in the LO frequency. For reference the results are also depicted for a retrieval without a shift in LO frequency for the case that the LO frequency is not retrieved (dashed black line) and that this frequency is retrieved (solid black line). The latter is obscured by the other retrieval results, indicating that the retrieval is almost not influenced by an uncertainty in this frequency.

expected in the stratosphere and consequently the retrieval error will then be $\approx 4 \%$. Expected uncertainties in the local oscillator signal (central frequency, shape, and width), the width of the field of view, and in case of $\mathrm{H}_{2}{ }^{18} \mathrm{O}$, also the sideband ratio have only limited impact on these retrievals. If the pointing error does not exceed $\approx 5$ arc min, this uncertainty is also of minor relevance. In fact, only the sideband ratio may induce a systematic shift in the HDO profile. For the estimated upper limit of 5\% in the knowledge of this ratio, the bias in the HDO profile is estimated to be less than $2.5 \%$, which is slightly smaller than the retrieval error. This means that isotopic water can be measured from a single limb scan down to the lower stratosphere with an accuracy and resolution that is sufficient to address the origin of stratospheric water and stratospheric water dynamics.

HDO can even be retrieved between 10-20 km probing the upper troposphere as well. With perfect knowledge of the instrument this can be done with a retrieval error of $1-6 \%$ and a FWHM of the averaging kernel of 1.5-2.5 km. However, this retrieval is deteriorated when pointing information has to be retrieved as well. In that case the retrieval error increases up to $\approx 35 \%$.

In that altitude range, the profile of $\mathrm{H}_{2}{ }^{18} \mathrm{O}$ becomes intermingled with the profile of the main water isotopologue hampering straightforward interpretation.

In conclusion $\mathrm{HDO}$ and $\mathrm{H}_{2}{ }^{18} \mathrm{O}$ can both be retrieved in the altitude range $20-37 \mathrm{~km}$ from a single limb scan. In addition HDO can also be retrieved between 10 and $20 \mathrm{~km}$, albeit with an enhance retrieval error. It is noted that the sideband ratio may cause a systematic error up to $2.5 \%$ in the case of HDO. TELIS can therefore address scientific issues pertaining to water dynamics in the upper troposphere and lower stratosphere. 
Acknowledgements. The authors would like to acknowledge Thomas Röckmann for fruitful discussions on isotopic water and its relevance to stratospheric research.

Edited by: G. Stiller

\section{References}

Anderson, G., Chetwynd, J., Clough, S., Shettle, E., and Kneizys, F.: AFGL atmospheric constituent profiles $(0-120 \mathrm{~km})$, Tech. Rep. AFGL-TR-86-0110, Air Force Geophysics Laboratory, Hanscom Air Force Base, Bedford, Mass., 1986.

Brewer, A.: Evidence for a world circulation provided by the measurements of helium and water vapor distribution in the stratosphere, Q. J. Roy. Meteorol. Soc., 75, 351-363, 1949.

Danielsen, E.: A dehydration mechanism for the stratosphere, Geophys. Res. Lett., 9, 605-608, 1982.

Franz, P. and Röckmann, T.: High-precision isotope measurements of H2-16O, H2-17O, H2-18O, and the Delta 17O-anomaly of water vapor in the southern lowermost stratosphere, Atmos. Chem. Phys., 5, 2949-2959, 2005,

http://www.atmos-chem-phys.net/5/2949/2005/.

Friedl-Vallon, F., Maucher, G., Seefeldner, M., Trieschmann, O., Kleinert, A., Lengel, A., Keim, C., Oelhaf, H., and Fischer, H.: Design and characterization of the balloon-borne michelson interferometer for passive atmospheric sounding (MIPAS-B2), Appl. Opt., 43, 3335-3355, 2004.

Hansen, P.: Analysis of discrete ill posed problems by means of the L-curve, SIAM Rev., 34, 561-580, 1992.

Holton, J. and Gettelman, A.: Horizontal transport and the dehydration of the stratosphere, Geophys. Res. Lett., 28, 2799-2802, 2001.

Hoogeveen, R., Yagoubov, P., de Lange, A., Selig, A., Koshelets, V., Ellison, B., and Birk, M.: Superconducting integrated receiver development for TELIS, Proc. SPIE, 5978, 440-450, 2005.

Johnson, D., Jucks, K., Traub, W., and Chance, K.: Isotopic composition of stratospheric water vapor: Implications for transport, J. Geophys. Res., 106, 12219-12226, 2001.

Koshelets, V., Ermakov, A., Filippenko, L., Lyudmila, V., Khudchenko, A., Kiselev, O., Sobolev, A., Alexander, S., Torgashin, M., Yagoubov, P., Hoogeveen, R., and Wild, W.: Superconducting integrated submillimeter receiver for TELIS, IEEE T. Appl. Supercon., 17, 336-342, doi:10.1109/TASC.2007.898622, 2007.

Liebe, H.: MPM-An atmospheric millimeter-wave propagation model, Int. J. Infrared and Millimeter Waves, 10, 631-650, 1989.

Moyer, E., Irion, F., Yung, Y., and Gunson, M.: ATMOS stratospheric deuterated water and implications for tropospherestratosphere transport, Geophys. Res. Lett., 23, 2385-2388, 1996.

Nassar, R., Bernath, P. F., Boone, C. D., Gettelman, A., McLeod, S. D., and Rinsland, C. P.: Variability in $\mathrm{HDO} / \mathrm{H}_{2} \mathrm{O}$ abundance ratios in the tropical tropopause layer, J. Geophys. Res., 112, D21305, doi:10.1029/2007JD008417, 2007.

Phillips, P.: A technique for the numerical solution of certain integral equations of the first kind, J. Assoc. Comput. Mach., 9, 84-97, 1962.

Read, W., Schwartz, M., Lambert, A., Su, H., Livesey, N., Daffer, W., and Boone, C.: The roles of convection, extratropical mix- ing, and in-situ freeze-drying in the Tropical Tropopause Layer, Atmos. Chem. Phys., 8, 6051-6067, 2008.

Ridal, M., Jonsson, A., Werner, M., and Murtagh, D. P.: A onedimensional simulation of the water vapor isotope HDO in the tropical stratosphere, J. Geophys. Res., 106, 32283-32294, 2001.

Rodgers, C. D.: Inverse Methods for Atmospheric Sounding: Theory and Practice, World Scientific, River Edge, N. J., 2000.

Rosenlof, K. H.: How Water Enters the Stratosphere, Science, 302, 1691-1692, 2003.

Rothman, L., Jacquemart, D., Barbe, A., Benner, D. C., Birk, M., Brown, L., Carleer, M., Jr., C. C., Chance, K., Coudert, L., Dana, V., Devi, V., Flaud, J.-M., Gamache, R., Goldman, A., Hartmann, J.-M., Jucks, K., Maki, A., Mandin, J.-Y., Massie, S., Orphal, J., Perrin, A., Rinsland, C., Smith, M., Tennyson, J., Tolchenov, R., Toth, R., Auwera, J. V., Varanasi, P., and Wagner, G.: The HITRAN 2004 molecular spectroscopic database, J. Quant. Spectrosc. Radiat. Transfer, 96, 139-204, 2005.

Sherwood, S. and Dessler, A.: On the control of stratospheric humidity, Geophys. Res. Lett., 27, 2513-2516, 2000.

Smith, J., Ackerman, A., Jensen, E., and Toon, O.: Role of deep convection in establishing the isotopic composition of water vapor in the tropical transition layer, Geophys. Res. Lett., 33, L06812, doi:10.1029/2005GL024078, 2006.

Steinwagner, J., Milz, M., von Clarmann, T., Glatthor, N., Grabowski, U., Höpfner, M., Stiller, G. P., , and Röckmann, T.: HDO measurements with MIPAS, Atmos. Chem. Phys., 7, 26012615, 2007, http://www.atmos-chem-phys.net/7/2601/2007/.

Tikhonov, A.: On the solution of incorrectly stated problems and a method of regularization, Dokl. Akad. Nauk SSSR, 151, 501$504,1963$.

Twomey, S.: On the numerical solution of Fredholm integral equations of the first kind by inversion of the linear system produced by quadrature, J. Assoc. Comput. Mach., 10, 97-101, 1963.

Urban, J., Lautié, N., Murtagh, D., Eriksson, P., Kasai, Y., Loßow, S., Dupuy, E., de La Noë, J., Frisk, U., Olberg, M., Flochmoën, E. L., and Ricaud, P.: Global observations of middle atmospheric water vapour by the Odin satellite, Planet. Space Sc., 55, 1093 1102, 2007.

Vleck, J. V. and Weisskopf, V.: On the Shape of CollisionBroadened Lines, Rev. Mod. Phys., 17, 227-236, 1945.

Webster, C. and Heymsfield, A.: Water Isotope Ratios D/H, 18O/16O, 17O/16O in and out of Cloud Map Dehydration Pathways, Science, 302, 1742-1745, 2003.

Weidner, F., Bösch, H., Bovensmann, H., Burrows, J., Butz, A., Camy-Peyret, C., Dorf, M., Gerilowski, K., Gurlit, W., Platt, U., von Friedeburg, C., Wagner, T., and Pfeilsticker, K.: Balloonborne limb profiling of UV/vis skylight radiances, $\mathrm{O}_{3}, \mathrm{NO}_{2}$, and BrO: technical set-up and validation of the method, Atmos. Chem. Phys., 5, 1409-1422, 2005, http://www.atmos-chem-phys.net/5/1409/2005/.

Worden, J., Bowman, K., Noone, D., Beer, R., Clough, S., Eldering, A., Fisher, B., Goldman, A., Gunson, M., Herman, R., Kulawik, S. S., Lampel, M., Luo, M., Osterman, G., Rinsland, C., Rodgers, C., Sander, S., Shephard, M., , and Worden, H.: Tropospheric Emission Spectrometer observations of the tropospheric $\mathrm{HDO} / \mathrm{H} 2 \mathrm{O}$ ratio: Estimation approach and characterization, J. Geophys. Res., 111, D16309, doi:10.1029/2005JD006606, 2006. Yagoubov, P., de Lange, G., Golstein, H., de Jong, L., de Lange, 
A., van Kuik, B., de Vries, E., Dercksen, J., Hoogeveen, R., Filippenko, L., Ermakov, A., and Koshelets, V.: Superconducting Integrated Receiver on Board TELIS, in: Proceedings of the 19th International Symposium on Space Terahertz Technology, SRON Netherlands Institute for Space Research, Groningen, the Netherlands, 2008.

Zahn, A., Franz, P., Bechtel, C., Grooss, J.-U., and Röckmann, T.: Modelling the budget of middle atmospheric water vapour isotopes, Atmos. Chem. Phys., 6, 2073-2090, 2006, http://www.atmos-chem-phys.net/6/2073/2006/.
Zelinger, Z., Barret, B., Kubát, P., Ricaud, P., Attie, J.-L., Urban, E. L. F. J., Murtagh, D., and Střižík, M.: Observation of HD-18O, $\mathrm{CH} 3 \mathrm{OH}$ and vibrationally-excited N2O from Odin/SMR measurements, Molec. Phys., 104, 2815-2820, 2006. 